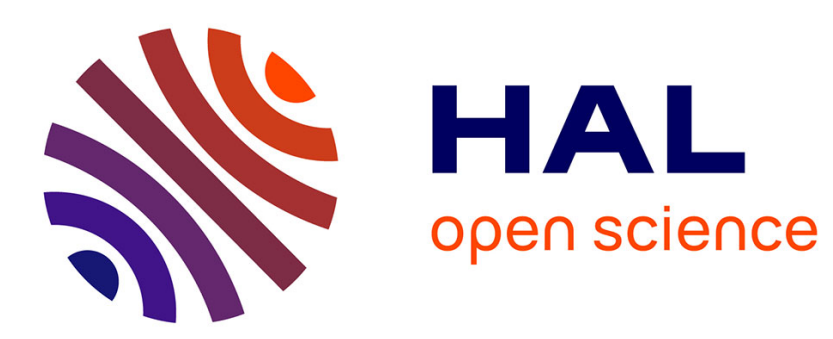

\title{
Dielectric Losses Considerations for Piezoelectric Energy Harvesting
}

\author{
Adrien Morel, Gaël Pillonnet, Yohan Wanderoild, Adrien Badel
}

\section{To cite this version:}

Adrien Morel, Gaël Pillonnet, Yohan Wanderoild, Adrien Badel. Dielectric Losses Considerations for Piezoelectric Energy Harvesting. Journal of Low Power Electronics, 2018, 14, pp.244 - 254. 10.1166/jolpe.2018.1562 . hal-01865139

\section{HAL Id: hal-01865139 \\ https://hal.science/hal-01865139}

Submitted on 23 Sep 2018

HAL is a multi-disciplinary open access archive for the deposit and dissemination of scientific research documents, whether they are published or not. The documents may come from teaching and research institutions in France or abroad, or from public or private research centers.
L'archive ouverte pluridisciplinaire HAL, est destinée au dépôt et à la diffusion de documents scientifiques de niveau recherche, publiés ou non, émanant des établissements d'enseignement et de recherche français ou étrangers, des laboratoires publics ou privés. 


\title{
Dielectric Losses Considerations for Piezoelectric Energy Harvesting
}

\author{
A. Morel ${ }^{1,2, *}$, G. Pillonnet ${ }^{1}$, Y. Wanderoild ${ }^{1}$, and A. Badel ${ }^{2}$ \\ ${ }^{1}$ Univ. Grenoble Alpes, CEA, LETI, F-38000 Grenoble, France \\ ${ }^{2}$ Univ. Savoie Mont Blanc, SYMME, F-74000 Annecy, France
}

(Received: 21 March 2018; Accepted: 27 March 2018)

\begin{abstract}
During the last decades, vibrations-based piezoelectric energy scavenging has been widely investigated as a way to replace or complement batteries to power sensor nodes in closed and confined environments. Piezoelectric ceramics exhibit conductive and dielectric losses. However, in most models used in energy harvesting, these losses are disregarded, as they appear to be relatively small in manufacturer datasheets. In this paper, after briefly introducing the origins of those losses, we experimentally prove that they are strongly dependent on the electric field in the material. This means that the datasheets data, obtained under low electric field characterizations, are not representative of the actual material losses under real-world vibrations. Thereafter, we prove that these losses limit the harvesting energy bandwidth, and we deduce the theoretical performance boundaries of piezoelectric harvesters. Finally, we study how harvesting energy on a linear resistive load is impacted by the losses in the piezoelectric material. Experimental results are in good agreement with our analysis and show that these losses should be taken into account in the electromechanical models in order to accurately predict the harvestable power from a linear piezoelectric harvester.
\end{abstract}

Keywords: Energy Harvesting, Piezoelectricity, Dielectric Losses, Electrical Interface, PZT, PMN-PT, PZN-PT.

\section{INTRODUCTION}

Nowadays, most sensors nodes are powered thanks to lithium and lithium-ion batteries. The main drawbacks of those systems is their limited longevity and their size, which make them hardly usable for powering abandoned, embedded, or biocompatible sensors nodes. Energy harvesting has been proposed as an alternative or a complement to battery, in order to make those sensors nodes autonomous and self-powered. Ambient energy can be found in the environment under the form of solar radiations, ${ }^{1}$ thermal gradients, ${ }^{2}$ air flows, ${ }^{3}$ electromagnetic radiations, ${ }^{4}$ biological organisms, ${ }^{5}$ and mechanical vibrations. ${ }^{6}$ Among the various energies type available in the environment, mechanical vibrations are of particular interest in closed, dark, confined places, where there are few solar radiations, nor thermal gradients. ${ }^{6}$

In order to convert the energy from a mechanical to an electrical form, three main transductions principles have been investigated as potential candidates: electromagnetic,

\footnotetext{
${ }^{*}$ Author to whom correspondence should be addressed.

Email: adrien.morel@cea.fr
}

electrostatic and piezoelectric. The choice of the transduction is mainly linked with the size of the harvester: for NEMS and MEMS applications, electrostatic harvesters are essentially the preferred choices. At mm-to$\mathrm{cm}$ scale, piezoelectric harvesters usually exhibit the highest power density. For larger harvesters, electromagnetic harvesters become the most appropriate. ${ }^{7,8}$ In this paper, we chose to focus our analysis on piezoelectric harvesters. It can however be noted that the analysis presented in the two last sections of this paper could be extended to electromagnetic harvesters, thanks to the dual nature of the electromagnetic/piezoelectric harvesters. ${ }^{9}$

A linear piezoelectric energy harvester (PEH) is usually made of a piezoelectric material deposited on a linear mechanical resonator, whose dynamic is governed by a second order linear differential equation. When this resonator is excited by an external force, a strain is applied on the piezoelectric material. Thanks to the piezoelectric effect, charges are generated and stored in the the material itself. The role of the electrical interface is to collect these charges in a way that maximizes the extracted energy from the mechanical structure to the electrical storage element. ${ }^{10}$ 
In most harvesters, the material used is made of hard ceramic exhibiting piezoelectric effect, such as lead zirconate titanate (PZT). ${ }^{11,12}$ The main advantages of PZT are its low production cost and its relatively important electromechanical coupling coefficient. ${ }^{13}$

Some works have proposed new electrical interfaces based on single crystals piezoelectric materials, such as lead magnesium niobate-lead titanate (PMN-PT) or lead zinc niobate-lead titanate (PZN-PT). ${ }^{14,15}$ Indeed, those materials exhibit higher $k_{31}$ than PZT, and can be used to design highly coupled harvesters exhibiting high energy performances and whose resonance frequency can be electrically adapted on a relatively large frequency band. ${ }^{16-18}$ However, some of those papers experimentally exhibited the influences of losses that were not predicted by the theoretical model and could be associated with dielectric losses. ${ }^{15,17}$

In this paper, we demonstrate that the dielectric losses, usually low in the materials datasheets, become important and non-negligible under high electric fields, which may usually be the case in real harvesting applications. Following this analysis, we propose a model for those dielectric losses, and, from this model, we derive new fundamental limits of energy harvesting from linear PEHs. Finally, the influences of these losses while harvesting energy from a resistive load is analyzed and experimentally validated.

\section{DIELECTRIC LOSSES ORIGINS}

\subsection{Dielectric Losses Modelling}

A piezoelectric material is made of two conductors of length $L$, width $W$, separated by a dielectric of depth $D$. An electric field $E$ in the piezoelectric material is generated due to the charges $Q$ and $-Q$ on the positive and negative electrodes respectively. Considering that the electric field is uniform inside the material, the capacitance $C$ of such piezoelectric plate can be derived using Gauss's law, and is given by (1).

$$
\left\{\begin{array}{l}
\oiint \vec{E} \cdot \vec{d} S=E L W=\frac{Q}{\varepsilon_{0} \varepsilon_{r}} \\
C=\frac{Q}{\left|\int_{0}^{D} E d z\right|}=\frac{\varepsilon_{0} \varepsilon_{r} L W}{D}=\frac{\varepsilon_{0} \varepsilon_{r} A}{D}
\end{array}\right.
$$

Where $\varepsilon_{0}$ is the permittivity of free space (8.85. $10^{-12} \mathrm{~F} \cdot \mathrm{m}^{-1}$ ) and $A$ is the electrode area. If the dielectric is considered imperfect and has a finite resistivity $\rho$, it presents an intrinsic resistance $R_{\text {cond }}=\rho D / A$. Hence, the energy losses associated with $R_{\text {cond }}$ are caused by the leakage current in the dielectric material. When the material is under a varying electric field, there are some additional dissipation of electromagnetic energy induced in the dielectric material itself, due to dipole rotations and oscillations. These losses, also known as dielectric losses, can be taken into account by considering a complex permittivity $\varepsilon_{r}^{*}=\varepsilon_{r}^{\prime}+j \varepsilon_{r}^{\prime \prime} \cdot{ }^{19}$ The piezoelectric material's admittance taking into account these two different losses is given by (2).

$$
Y_{p}=j \omega \frac{\varepsilon_{0} \varepsilon_{r}^{\prime} A}{D}+\omega \frac{\varepsilon_{0} \varepsilon_{r}^{\prime \prime} A}{D}+\frac{A}{\rho D}=j \omega C_{p}+\frac{1}{R_{\varepsilon}}+\frac{1}{R_{\text {cond }}}
$$

This admittance expression (2) includes a real and an imaginary part. Hence we can consider the piezoelectric material electrical model as a capacitance $C_{p}$ (modeling its ability to store electric charges) in parallel with two resistances $R_{\varepsilon}$ and $R_{\text {cond }}$ modeling respectively the energy losses due to the thermal agitation in the dielectric material, and due to the leakage current in the material, as shown in Figure 1. Of course, this electrical model remains a mathematical representation of the behavior of the dielectric material. The ratio $\tan (\delta)$, commonly called "loss tangent," is then defined as (3): 19

$$
\tan (\delta)=\frac{1}{\left(R_{\varepsilon} / / R_{\text {cond }}\right) C_{p} \omega}=\frac{\sigma}{\varepsilon_{0} \varepsilon_{r}^{\prime} \omega}+\frac{\varepsilon_{r}^{\prime \prime}}{\varepsilon_{r}^{\prime}}
$$

With $\sigma$ the conductivity of the dielectric material, defined as $1 / \rho$.

\subsection{Influences of the Voltage Frequency on the Dielectric Losses}

From (3), it can be noticed that the loss tangent $\tan (\delta)$ is the sum of two terms:

- $\sigma /\left(\varepsilon_{0} \varepsilon_{r}^{\prime} \omega\right)$, due to the finite resistivity of the dielectric material, which varies with the frequency of the voltage across the piezoelectric material electrodes.

- $\varepsilon_{r}^{\prime \prime} / \varepsilon_{r}^{\prime}$ which is due to the thermal agitation in the dielectric material, and is, in another hand, not dependent on the frequency of the voltage across the piezoelectric material electrodes.

In order to study how $\tan (\delta)$ behaves with frequency variations, two piezoelectric materials have been characterized thanks to an impedance analyzer. Figure 2 shows the module and phase of the admittance of a PZT material plate while Figure 3 shows the the same figure for a PMN-PT material plate.

The phase of the admittance $\phi_{Y}$ is directly linked to the loss tangent $\tan (\delta)$ by relation (4).

$$
\phi_{Y}=\frac{180}{\pi} \arctan (1 / \tan (\delta))
$$

We can observe that $\phi_{Y}$ remains relatively constant for any frequency $f \in[100 \mathrm{~Hz}, 200 \mathrm{~Hz}]$ for the PMN-PT material and $f \in[50 \mathrm{~Hz}, 500 \mathrm{~Hz}]$ for the PZT material. It means that $\tan (\delta)$ is constant as well on these frequency ranges. We can conclude that the main contribution to $\tan (\delta)$ is constant with the frequency and is thus given by $\varepsilon_{r}^{\prime \prime} / \varepsilon_{r}^{\prime}$. This physically means that the conduction losses in the dielectric material are negligible in comparison with the dielectric losses. We have been able to determine as well the three components of the piezoelectric electrical 

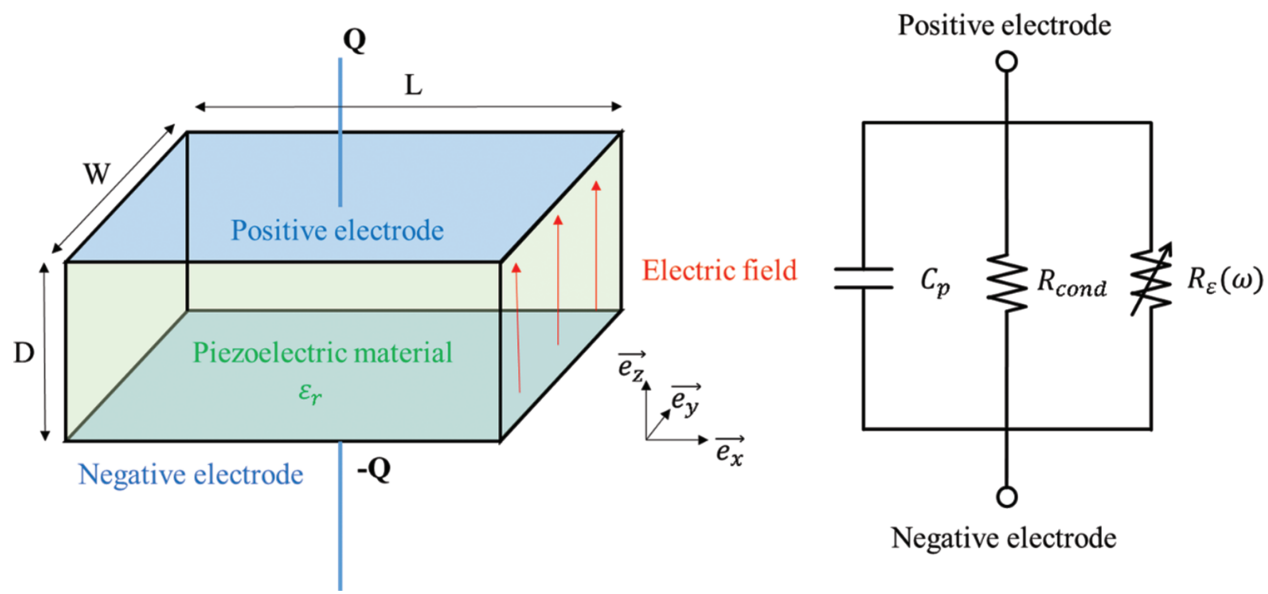

Fig. 1. Piezoelectric material plate representation and its associated equivalent electrical model.

model $C_{p}, R_{\varepsilon}, R_{\text {cond }}$ by fitting the theoretical admittance expression given by (3) to experimental data shown on Figures 2 and 3 for $f \in[100 \mathrm{~Hz}, 200 \mathrm{~Hz}]$. These parameters values have been summarized in Table I.

We can observe that $R_{\text {cond }}$ is way more important than $R_{\varepsilon}$. This confirms that the losses in the dielectric material are mainly due to the dielectric losses, and not to the conduction losses, leading to an almost invariant loss tangent over the considered frequency range. ${ }^{20}$ If we neglect $R_{\text {cond }}$ in (3), we obtain the well-known expression of $\tan (\delta)$ given by $(5)$, which is frequency independent. ${ }^{21}$

$$
\tan (\delta) \approx \frac{\varepsilon_{r}^{\prime \prime}}{\varepsilon_{r}^{\prime}}
$$

From now on, we will consider the expression (5) to be true, and only take into account the losses due to the thermal agitation in the dielectric material.

\subsection{Influences of the Electric Field on the Dielectric Losses}

Some recent piezoelectric materials data from papers and manufacturers datasheets have been gathered and
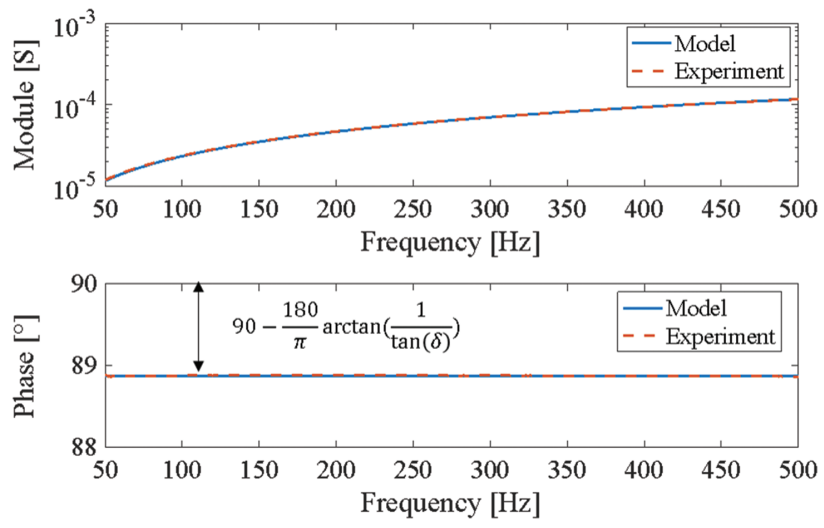

Fig. 2. Experimental admittance analysis of a PZT material and fitted analytical model. summarized in Figure 4, with respect to their coupling coefficients $k_{31}$ and their quality factor $Q_{\text {diel }}=1 / \tan (\delta)$. From manufacturer datasheets and prior art, PZN-PT materials appear to present highest $k_{31}$, followed by PMN-PT, soft ceramics, then hard ceramics. The dielectric losses tangent of all those materials, even if they appear to be a bit more important for soft ceramics, remain relatively small (always lower than 2 or $3 \%$ ).

Those losses are however characterized under low electric fields. In order to see whether or not the dielectric losses remain small under higher electric fields, we characterized the dielectric losses of two different piezoelectric materials under various AC electric fields levels. To do so, we used the experimental setup shown in Figure 5.

The piezoelectric materials used were a PZT ceramic having a width of $200 \mu \mathrm{m}$, and a PMN-PT material having a width of $500 \mu \mathrm{m}$. They have been excited from 0 to $210 \mathrm{~V}$ thanks to an Agilent B2962A power source. The voltage difference across the sensing resistor $R_{\text {sense }}$ gives us the current $I_{R}$ flowing through the piezoelectric harvester. Since we know both $I_{R}$ and the voltage across the
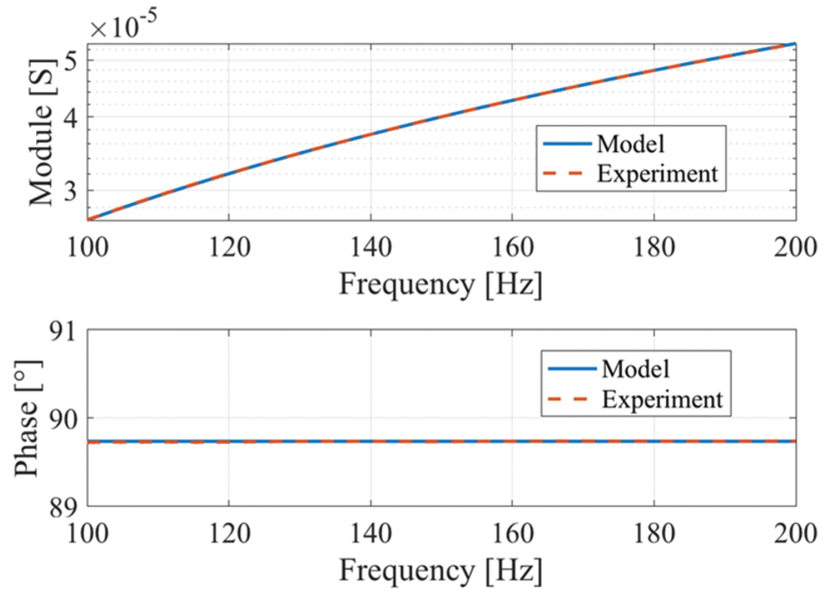

Fig. 3. Experimental admittance analysis of a PMN-PT material and fitted analytical model.

J. Low Power Electron. 14, 244-254, 2018 
Table I. Piezoelectric energy harvester's characteristics.

\begin{tabular}{|c|c|c|c|}
\hline \multirow[b]{2}{*}{ Parameters } & \multicolumn{2}{|c|}{ Piezoelectric material } & \multirow[b]{2}{*}{ Units } \\
\hline & PZT & PMN-PT & \\
\hline$C_{p}$ & 37 & 42 & $\mathrm{nF}$ \\
\hline$R_{\varepsilon} \omega$ & 1.36 & 5.150 & $\mathrm{G} \Omega \cdot \mathrm{rad}$ \\
\hline$R_{\text {cond }}$ & 4246 & 16000 & $\mathrm{G} \Omega$ \\
\hline $\tan (\delta)$ & $\approx 1.97 \%$ & $\approx 0.46 \%$ & N/A \\
\hline
\end{tabular}

piezoelectric material, $V_{2}$, we can get the phase between those two signals, and their ratio. Thus, we can easily determine the values of the electrical models $R_{\varepsilon}$ and $C_{p}$, for various voltage frequency and amplitude.

$$
\left\{\begin{array}{l}
|Y|=\frac{\left|V_{1}-V_{2}\right|}{R_{\text {sense }}\left|V_{2}\right|}=\sqrt{\frac{1}{R_{\varepsilon}^{2}}+C_{p}^{2} \omega^{2}} \\
\arg (Y)=\phi\left(\frac{V_{1}-V_{2}}{R_{\text {sense }}}, V_{2}\right)=\arctan \left(R_{\varepsilon} C_{p} \omega\right)
\end{array}\right.
$$

$\tan (\delta)$ has been characterized with various voltage amplitude, from few $\mathrm{mVs}$ to 10th of volts, and its evolution can be seen on Figure 6.

From those tests, we can notice that when the electric field is larger, the dielectric losses increase as well. This is consistent with the model proposed by Yang et al. in

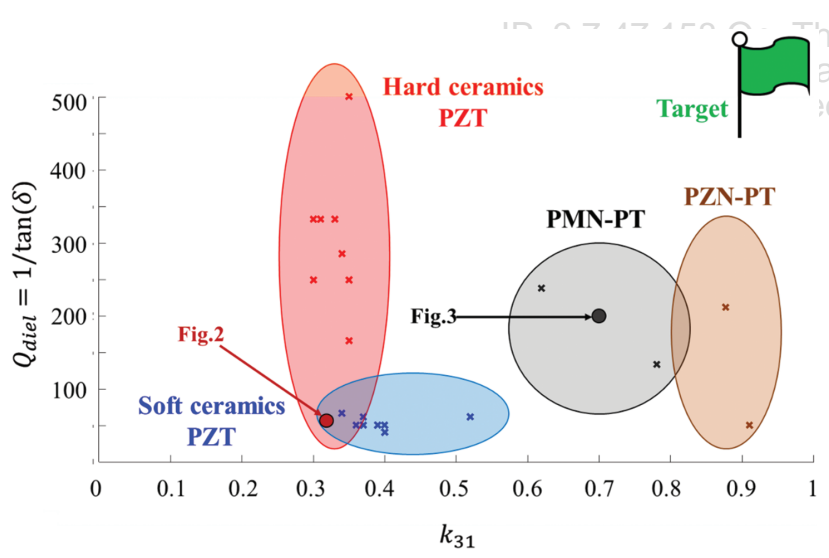

Fig. 4. Coupling $k_{31}$ and loss tangent $\tan (\delta)$ of various materials exhibiting piezoelectric properties characterized under small electric fields, with data from Refs. [16, 20-25].

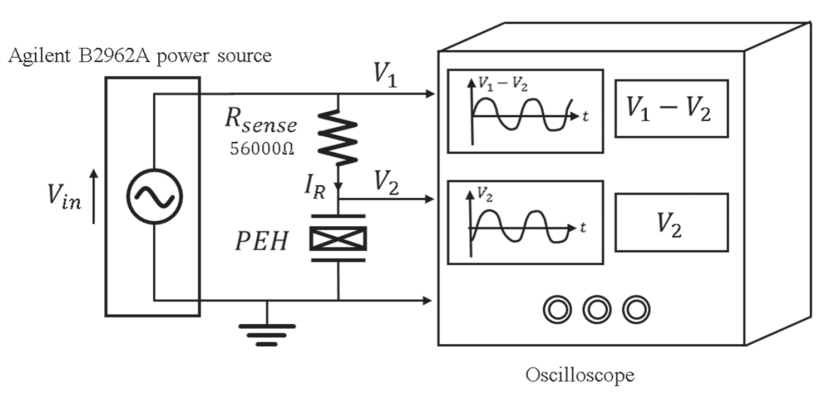

Fig. 5. Experimental setup for exhibiting the non-linear behavior of the dielectric losses.

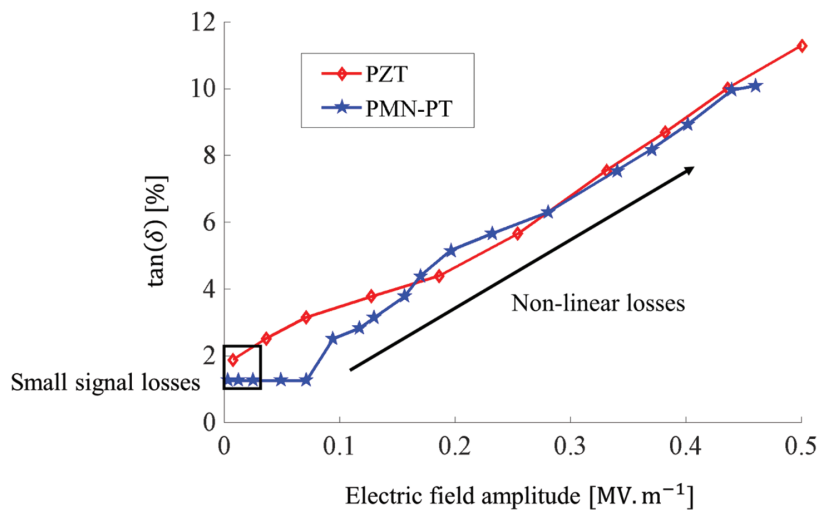

Fig. 6. Evolution of $\tan (\delta)$ as a function of the AC electric field amplitude applied on the piezoelectric materials.

Ref. [24] who considered these losses nonlinearities as a polynomial function of the electric field, as reformulated by (7). In our case, as shown in Figure $6, \tan (\delta)$ can be approximated as a first order polynomial function of $E$ with $a_{0} \approx 0.01$ and $a_{1} \approx 2 \cdot 10^{-7}$.

$$
\tan (\delta) \approx \sum_{i=0}^{\infty} a_{i} E^{i} \approx a_{0}+a_{1} E
$$

Thus, under important mechanical excitations leading to high electric field in the piezoelectric material, these dielectric losses have an increasingly impact on the harvestable energy. For identical converted power and same material dimension, strongly coupled piezoelectric material such as PMN-PT or PZN-PT exhibit higher electric field than PZT materials, because of their smaller piezoelectric capacitance $C_{p}$ due to their lower $\varepsilon_{r} \cdot{ }^{24,26}$ Hence, even though single crystals materials do not exhibit high dielectric losses under low electric fields, they may however exhibit important losses under high electric fields, which is the case under real world vibrations.

\section{BOUNDARIES OF LINEAR ENERGY HARVESTING}

In order to study the influences of these losses in the context of energy harvesting, let's consider a piezoelectric energy harvester made of a linear oscillator modelled by a linear single degree of freedom differential equation. A piezoelectric material is deposited upon this mechanical oscillator, as shown in Figure 7. When the harvester is excited under an external force $F$, a mechanical strain is applied on the material. Thanks to the direct piezoelectric effect, electrical charges are consequently generated in the piezoelectric material. The constitutive equations of such linear coupled system can be expressed by (8). ${ }^{18,27}$

$$
\left\{\begin{array}{l}
F=M \gamma=M \ddot{x}+D \dot{x}+K_{\mathrm{sc}} x+\alpha v_{p} \\
i_{p}=\alpha \dot{x}-C_{p} \dot{v}_{p}-v_{p} / R_{\varepsilon}
\end{array}\right.
$$

Where $M, K_{\mathrm{sc}}, \alpha$ stand for the effective mass of the harvester, its effective short-circuit stiffness, and its 

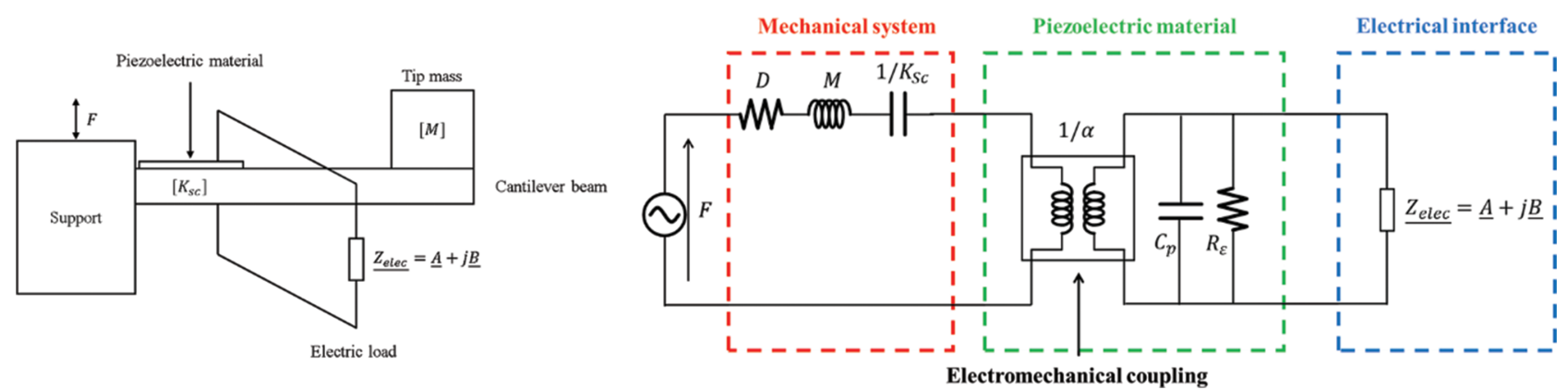

Fig. 7. Piezoelectric energy harvester representation and its associated electrical equivalent model.

force coefficient respectively. $D$ represents the mechanical damping, $\gamma$ the acceleration of the excitation, $x$ the mass displacement, and $i_{p}$ the current going into the electrical interface. The electrical equivalent model of (8) is shown on Figure 7.

In order to study this model, some normalized variables have been proposed in previous works, as shown in Table II. $\omega_{0}$ is the short-circuit angular resonance frequency of the harvester, $\Omega_{m}$ is its normalized resonance frequency, $k_{m}^{2}$ its normalized squared coupling coefficient, and $\xi_{m}$ is the normalized mechanical damping.

From this model, two conditions must be satisfied in order to maximize the harvested energy:

- The damping induced by the electrical interface should be equal to the mechanical damping. ${ }^{27,28} .47 .158 \mathrm{On}$ : Tht - The electromechanical system's resonance frequency should be equal to the input vibrations frequency. ${ }^{27}$

$$
\frac{\underline{v}_{p}}{\underline{i}_{p}}=\underline{Z}_{\mathrm{elec}}=\underline{A}+j \underline{B}
$$

Due to the filtering effect of the mechanical resonator, we can consider that only the first harmonic of the piezoelectric voltage will influence the dynamics of the electromechanical system. Thus, based on this assumption, the electrical interface can always be modelled as a complex impedance $\underline{Z}_{\text {elec }}$ which presents a real part $\underline{A} \in \mathbb{R}^{+*}$ and an imaginary part $\underline{B} \in \mathbb{R}^{27,29}$ If there is no restrictions on the electrical interface (meaning that $\underline{A}$ and $\underline{B}$ can take any value in their definition domains), and if the piezoelectric material is lossless $\left(\tan (\delta)=\varepsilon_{r}^{\prime \prime}=0, R_{\text {cond }} \rightarrow \infty\right.$, $R_{\varepsilon} \rightarrow \infty$ ), then the two conditions above can always be met, and the maximum harvested power given by (10) can

Table II. Normalized variables expressions.

\begin{tabular}{lcc}
\hline Normalized parameters & Expressions & Domains \\
\hline$\omega_{0}$ & $\sqrt{K_{\mathrm{sc}} M^{-1}}$ & Mechanical \\
$\xi_{m}$ & $\frac{D}{2} \sqrt{K_{\mathrm{sc}}^{-1} M^{-1}}$ & Mechanical \\
$\Omega_{m}$ & $\omega / \omega_{0}$ & Vibrational \\
$k_{m}^{2}$ & $k^{2} /\left(1-k^{2}\right)=\alpha^{2} /\left(K_{\mathrm{sc}} C_{p}\right)$ & Material \\
\hline
\end{tabular}

be reach for any vibration frequency, coupling or mechanical damping. ${ }^{30,31}$

$$
\left(P_{\max }\right)_{\text {lossless }}=\frac{|M \gamma|^{2}}{8 D}
$$

However, if the dielectric losses are taken into account, this limit becomes a function of the losses themselves, but also of the vibration angular frequency, mechanical damping, and electromechanical coupling. From (8) and (9), it can be shown that this maximum is expressed by (11). ${ }^{27}$

$$
\begin{aligned}
& \left(P_{\max }\right)_{\text {lossy }}=\frac{|M \gamma|^{2}}{8 D} /\left(1+\frac{2 \sqrt{M K_{\mathrm{sc}}} M}{2 D \alpha^{2} R_{\varepsilon}}\left(\frac{4 D^{2}}{K_{\mathrm{sc}} M}\right.\right. \\
& \left.\left.201820: 49+M \frac{\left(M \omega^{2} / K_{\mathrm{sc}}-1\right)^{2}}{K_{\mathrm{sc}} \omega^{2}}\right)\right)
\end{aligned}
$$

Normalizing (11) with respect to the maximum harvested power with a lossless piezoelectric material, and replacing the model parameters by their normalized counterparts (Table II), we can get the expression (12).

$$
\begin{aligned}
\eta & =\frac{\left(P_{\max }\right)_{\text {lossy }}}{\left(P_{\max }\right)_{\text {lossless }}} \\
& =\frac{1}{1+\tan (\delta) \Omega_{m} /\left(2 k_{m}^{2} \xi_{m}\right)\left(4 \xi_{m}^{2}+\left(\Omega_{m}^{2}-1\right)^{2} / \Omega_{m}^{2}\right)}
\end{aligned}
$$

With $\eta \in[0,1]$, the normalized harvested power boundary which gives an information about how much the dielectric losses influence the harvested energy boundaries. For a lossless dielectric material $(\tan (\delta)=0), \eta$ will be equal to unity. In another hand, if the dielectric losses are very important $(\tan (\delta) \rightarrow \infty), \eta$ will tend toward zero. An interesting insight given by (12) is that $\eta$ is only dependent on three parameters: the normalized mechanical damping $\xi_{m}$, the normalized vibration frequency $\Omega_{m}$, and the ratio of the dielectric loss tangent over the squared coupling coefficient $\tan (\delta) / k_{m}^{2}$. It means that a higher coupling coefficient will reduce the influences of the dielectric loss tangent on the harvested energy boundary. Figure 8 shows the evolution of $\eta$ in the plane $\left(\tan (\delta) / k_{m}^{2} ; \Omega_{m}\right)$.

From Figure 8 we can conclude the following:

- A higher loss tangent implies a smaller harvestable spectrum of energy. 


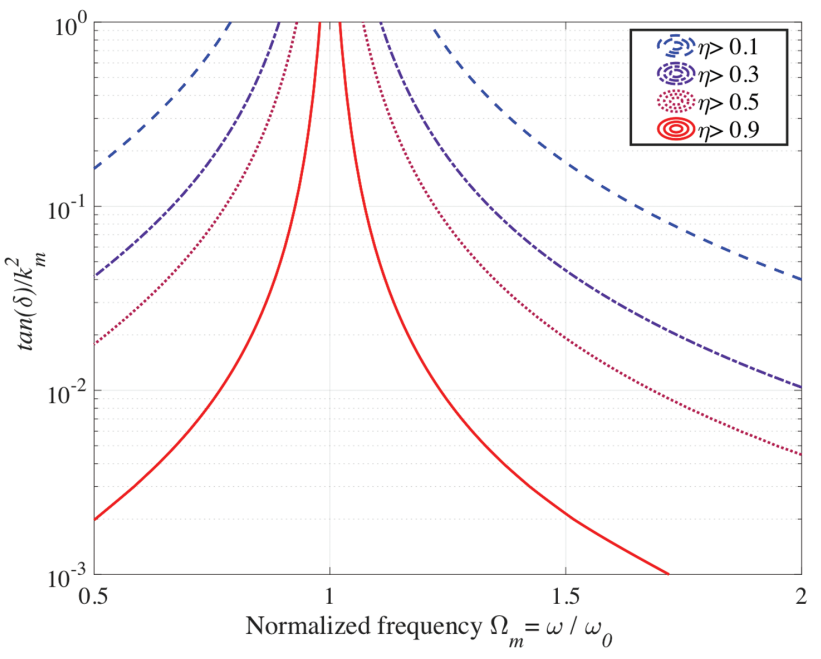

Fig. 8. Normalized harvested power boundary $\eta$ in the plane $\left(\tan (\delta) / k_{m}^{2} ; \Omega_{m}\right)$ with $\xi_{m}=0.01$.

- A higher $k_{m}^{2}$ implies a higher harvestable spectrum of energy.

- The dielectric losses are minimized when the vibration frequency matches the short-circuit resonance frequency of the PEH. Consequently, the influences of the dielectric losses are increased as the vibrations frequency get farther from the short-circuit resonance frequency.

When the vibration frequency matches the harvester resonance frequency, (12) can be greatly simplified, and the expression of $\eta$ can be given in this case by (13). This expression has been computed as a function of $\tan (\delta) / k_{m}^{2}$ for various mechanical damping, as shown in Figure 9. This figure shows that a higher mechanical damping leads to higher dielectric losses at resonance.

$$
\eta_{\text {resonance }}=\frac{1}{1+\left(2 \tan (\delta) / k_{m}^{2}\right) \xi_{m}}
$$

The normalized harvesting bandwidth of a $\mathrm{PEH}$ is given by the frequency range $\Delta \Omega$ where the harvested power is

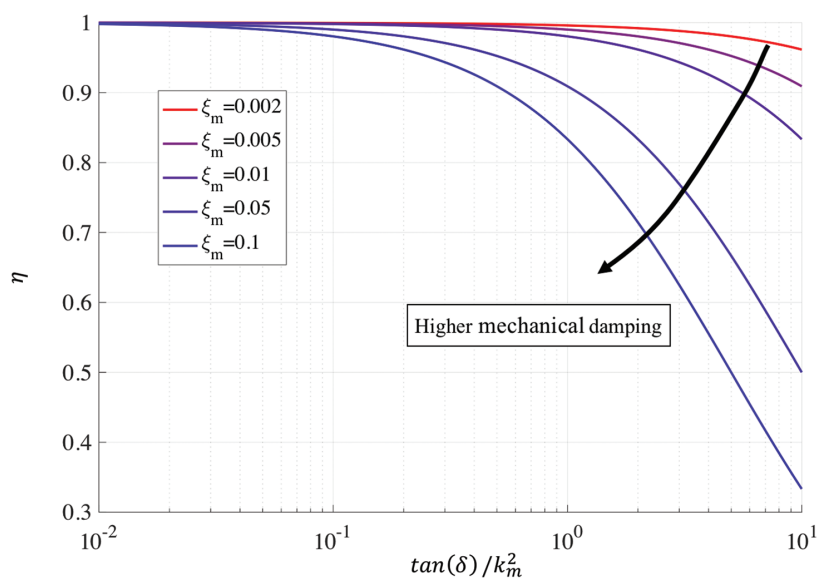

Fig. 9. Normalized harvested power boundary $\eta$ at resonance $\left(\Omega_{m}=1\right)$ with various dielectric losses, coupling, and mechanical damping.

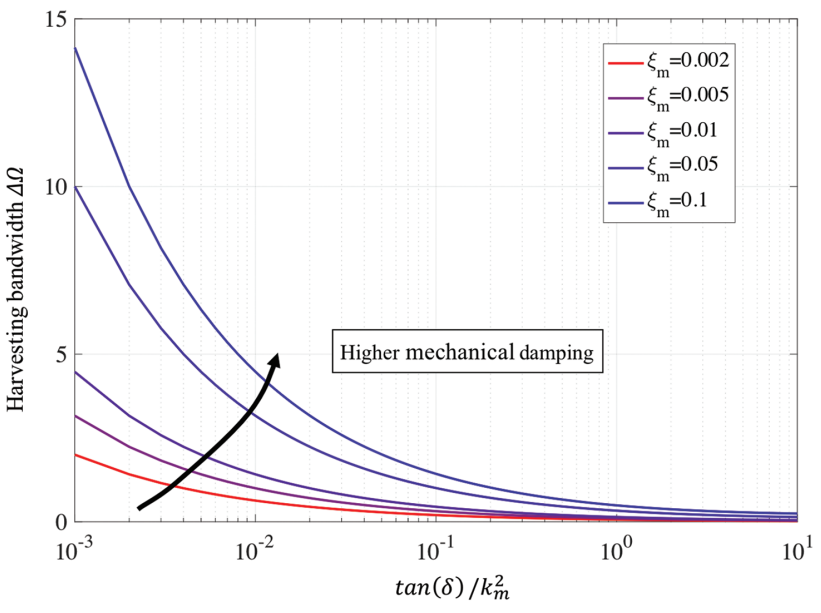

Fig. 10. Normalized harvesting bandwidth $\Delta \Omega$ with various dielectric losses, coupling, and mechanical damping.

higher than $50 \%$ of the maximum harvested power. From (12), we can derive the maximum $\Delta \Omega$ that can be reach for a particular linear $\mathrm{PEH}$, which is no longer unbounded.

$$
\Delta \Omega_{\max } \approx \sqrt{4 \xi_{m}^{2}+\frac{2 \xi_{m} k_{m}^{2}}{\tan (\delta)}}
$$

(14) has been computed as a function of $\tan (\delta) / k_{m}^{2}$ for various $\xi_{m}$, as shown in Figure 10. We can observe that a higher mechanical damping leads to a greater harvesting bandwidth.

In this part, we studied how the dielectric losses impact the boundaries of the harvested energy from linear energy harvesters in term of maximal power and bandwidth. These boundaries are a theoretical limitation that could be reach only with a perfect impedance matching realization. It means that the bandwidth improvement that could be reach with a perfect impedance matching and electrical tuning is somehow limited by the dielectric losses.

\section{DIELECTRIC LOSSES INFLUENCES ON LINEAR HARVESTING INTERFACES}

The previous analysis focused on the energy harvesting boundary with a perfectly matched electrical load. However, in most real cases, this impedance matching requires a highly inductive load reaching 100th of Henrys, which is obviously unpractical. ${ }^{27}$ In this part, we will focus on the influences of the dielectric losses on a simpler linear electrical interface made of a resistive load.

\subsection{Analytical Formulation}

The harvested power on a resistive load $R_{\text {load }}$ can be expressed by (15).

$$
P=\frac{v_{p_{m}}^{2}}{2 R_{\mathrm{load}}}
$$

Where $v_{p_{m}}$ is the magnitude of the piezoelectric voltage. The piezoelectric voltage can be found, solving the second 
equation of the system (8) in the frequency domain. Its expression is given by (16).

$$
\begin{aligned}
v_{p_{m}} & =\frac{\alpha X_{m} \omega R_{p} R_{\text {load }}}{\sqrt{\left(R_{p}+R_{\text {load }}\right)^{2}+\left(R_{\text {load }} C_{p} R_{p} \omega\right)^{2}}} \\
& =\frac{\alpha X_{m}}{C_{p}} \frac{1}{\sqrt{\left(r^{-1} \Omega_{m}^{-1}+\tan (\delta)\right)^{2}+1}}
\end{aligned}
$$

With $X_{m}$ the magnitude of the displacement $x$ and $r$ the normalized electrical load resistance whose expression is given by (17).

$$
r=R_{\text {load }} C_{p} \omega_{0}
$$

From the first equation of (8) in the Fourier domain, the expression of the displacement magnitude can be expressed as:

$$
\left\{\begin{array}{l}
X_{m}=\frac{|M \gamma|}{\sqrt{\left(-\omega^{2} M+K_{\mathrm{sc}}+A\right)^{2}+((D+B) \omega)^{2}}} \\
B=\frac{\alpha^{2}}{C_{p} \omega} \frac{\left(r^{-1} \Omega_{m}^{-1}+\tan (\delta)\right)}{1+\left(r^{-1} \Omega_{m}^{-1}+\tan (\delta)\right)^{2}} \\
A=\frac{\alpha^{2}}{C_{p}} \frac{1}{1+\left(r^{-1} \Omega_{m}^{-1}+\tan (\delta)\right)^{2}}
\end{array}\right.
$$

Thus, from (15), (16) and (18), the normalized power harvested on a linear resistance while taking into account the dielectric losses in the piezoelectric material can be determined, as expressed by (19).

$$
\begin{aligned}
P_{\text {norm }}= & \frac{P}{P_{\max _{\text {lossless }}}} \\
& =\left(\left[\frac{8 k_{m}^{2} \xi_{m}}{1+\left(r^{-1} \Omega_{m}^{-1}+\tan (\delta)\right)^{2}}\right]\right) \\
& /\left(r \left[\left(1-\Omega_{m}^{2}+\frac{k_{m}^{2}}{1+\left(r^{-1} \Omega_{m}^{-1}+\tan (\delta)\right)^{2}}\right)^{2}\right.\right. \\
& \left.\left.+\left(2 \Omega_{m} \xi_{m}+k_{m}^{2} \frac{\left(r^{-1} \Omega_{m}^{-1}+\tan (\delta)\right)}{1+\left(r^{-1} \Omega_{m}^{-1}+\tan (\delta)\right)^{2}}\right)^{2}\right]\right)
\end{aligned}
$$

Applying this relation on the piezoelectric harvester used in the experimental validation part (whose characteristics are summarized in Table III), we can see how the dielectric losses influences the harvested energy frequency

Table III. Piezoelectric harvester characteristics (under very small vibrations of $0.01 \mathrm{G}$ ).

\begin{tabular}{lcc}
\hline Parameters names & Parameters values & Units \\
\hline$C_{p}$ & 2 & $\mathrm{nF}$ \\
$\tan (\delta)$ & 0.1 & $\%$ \\
$\omega_{0}$ & 348.4 & $\mathrm{rad} \cdot \mathrm{s}^{-1}$ \\
$\xi_{m}$ & 0.0135 & - \\
$k_{m}^{2}$ & 0.39 & - \\
\hline
\end{tabular}

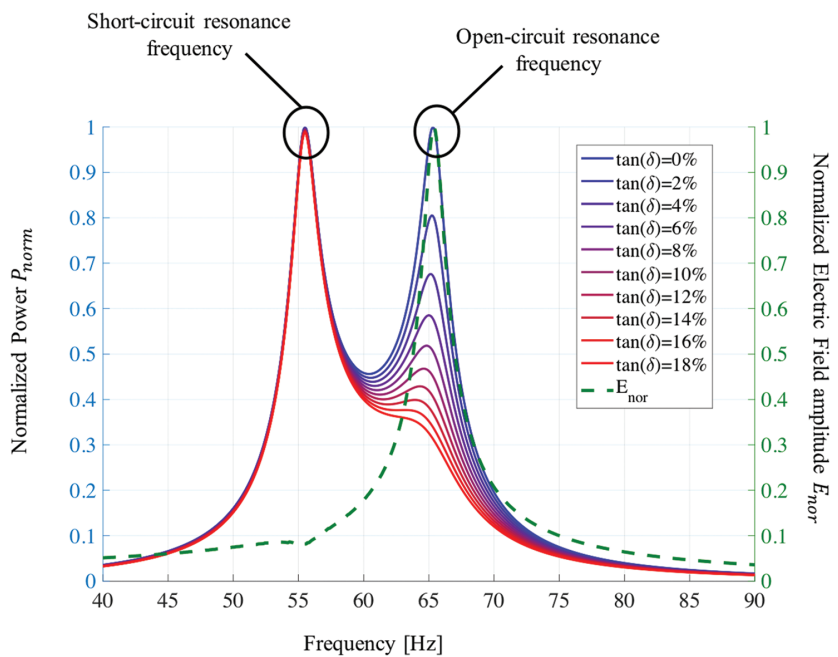

Fig. 11. Frequency responses of a highly coupled PEH (Table III) connected to an optimal resistive load, with various loss tangent values.

responses. Figure 11 shows the harvested energy on an optimized (for each frequency) resistive load with various loss tangent values. The harvested power frequency response of a highly coupled harvester on a resistive load exhibits two power peaks: one corresponding to the opencircuit resonance frequency of the harvester, and one corresponding to the short-circuit resonance frequency of the harvester. If we consider a lossless piezoelectric material, the two power peaks should reach the same value. ${ }^{31,32}$ However, because of the dielectric losses, the open-circuit resonance peak would largely decrease, while the shortcircuit resonance peak would almost remain unchanged. This is mainly because the optimal piezoelectric voltage (the voltage maximizing the energy extraction) around the short-circuit resonance frequency is inherently low, while it becomes greater around the open-circuit resonance frequency, leading to greater electric field and thus greater dielectric losses. This analysis is confirmed by the normalized AC electric field amplitude $E_{\text {nor }}=E / E_{\max }$, which is computed in green on Figure 11. This electric field is indeed maximal around the second power peak corresponding to the PEH open-circuit resonance frequency.

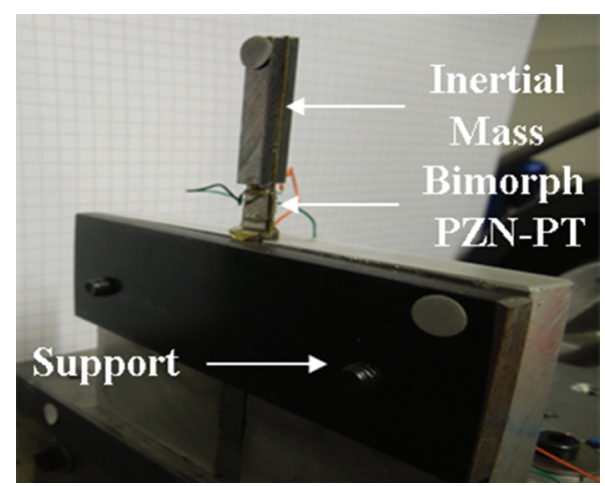

Fig. 12. Piezoelectric harvester made with two plates of PZN-PT. 


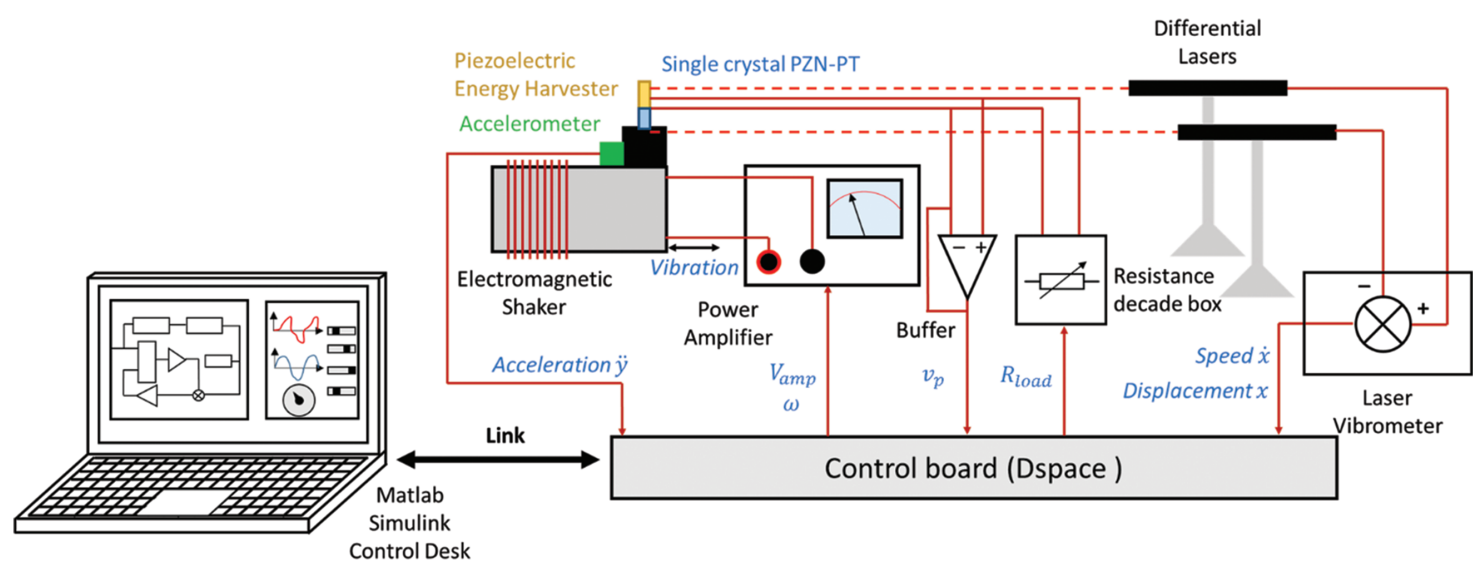

Fig. 13. Setup used for automatically collecting the harvested power data using a variable resistive load.

\subsection{Experimental Validation}

We conducted experiments with the PZN-PT piezoelectric harvester shown on Figure 12, whose characteristics have been summarized in Table III. We placed this piezoelectric harvester on an electromagnetic shaker and
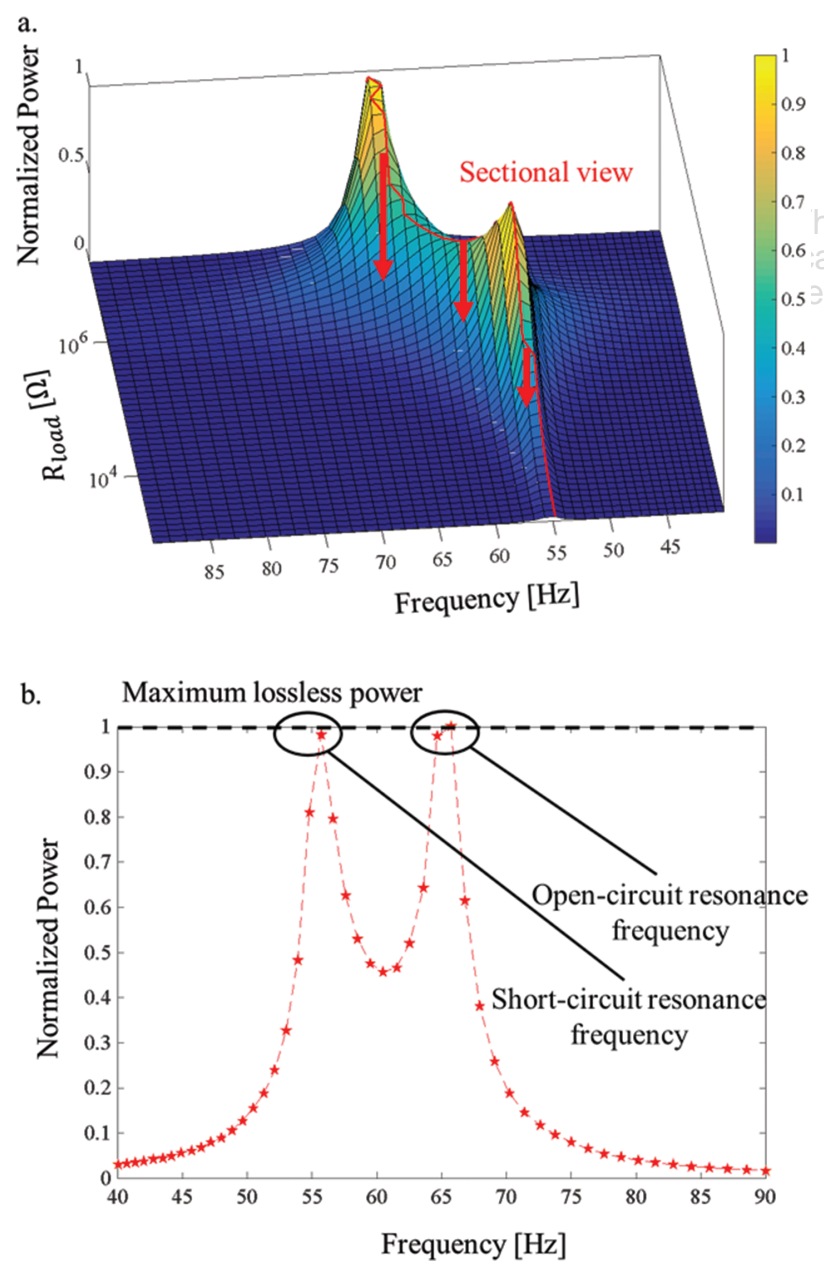

Fig. 14. Experimental harvested power (a) in the $\left(R_{\text {load }}, f\right)$ plane and (b) as a function of the frequency $f$ with an optimal $R_{\text {load }}$ maximizing the harvested power, with $\tan (\delta) \approx 0.1 \%$ and $\gamma=0.01 \mathrm{G}$. its electrodes has been connected to a programmable resistance decade box. Thanks to a dSpace program, the excitation frequency $\omega$, acceleration amplitude $|\gamma|$, and the resistive load $R_{\text {load }}$ have been consecutively changed,

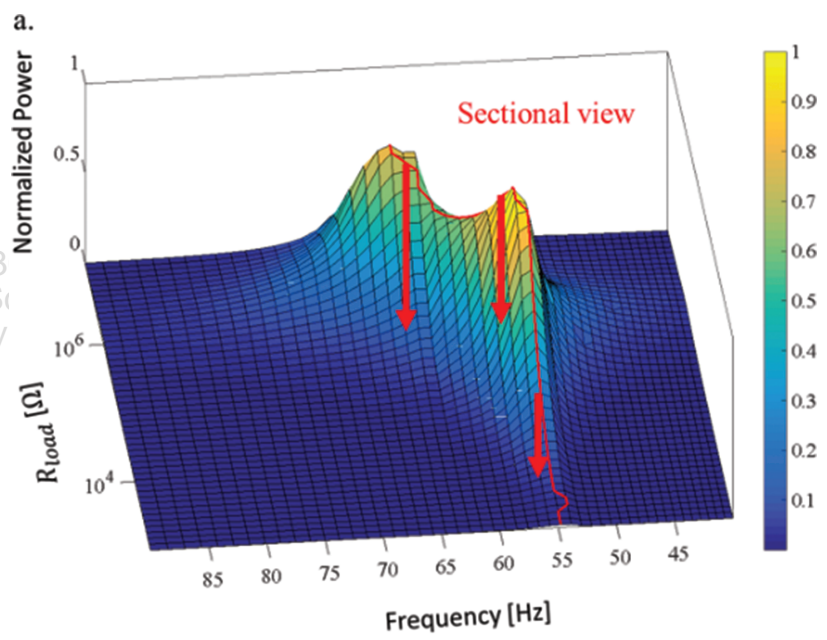

b. Maximum lossless power

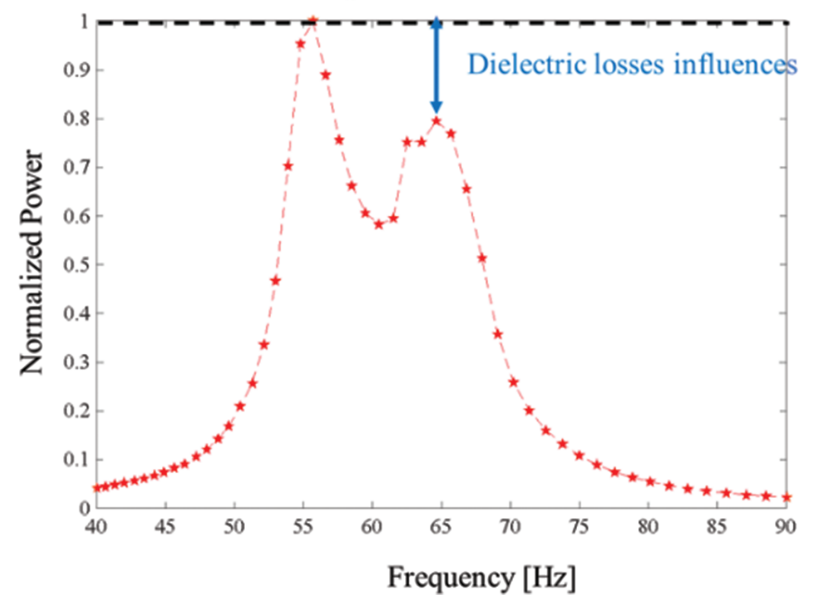

Fig. 15. Experimental harvested power (a) in the $\left(R_{\text {load }}, f\right)$ plane and (b) as a function of the frequency $f$ with an optimal $R_{\text {load }}$ maximizing the harvested power, with $\tan (\delta) \approx 5 \%$ and $\gamma=0.05 \mathrm{G}$. 
and the voltages, displacement, and acceleration waveforms have been automatically stored in a computer for each parameter configuration. The experimental setup is described on Figure 13.

From the voltage waveforms, we computed the experimental power on a resistive load in the plane $\left(R_{\text {load }}, f\right)$, for various excitation amplitude.

Figure 14 shows the experimental harvested power on a resistive load under a very small mechanical excitation of $0.01 \mathrm{G}$. Under this excitation, the electric field in the piezoelectric material remains small, and the dielectric losses are almost negligible. As predicted in the theoretical part, the harvested power reaches its maximum power point for two different frequencies which correspond to the $\mathrm{PEH}$ short-circuit and open-circuit resonance frequencies.

Figure 15 also shows the experimental harvested power on a resistive load, but with a slightly more important mechanical excitation of $0.05 \mathrm{G}$. Due to the higher strain, the piezoelectric material is under a higher electric field, which, as detailed in the first part of this paper, leads to
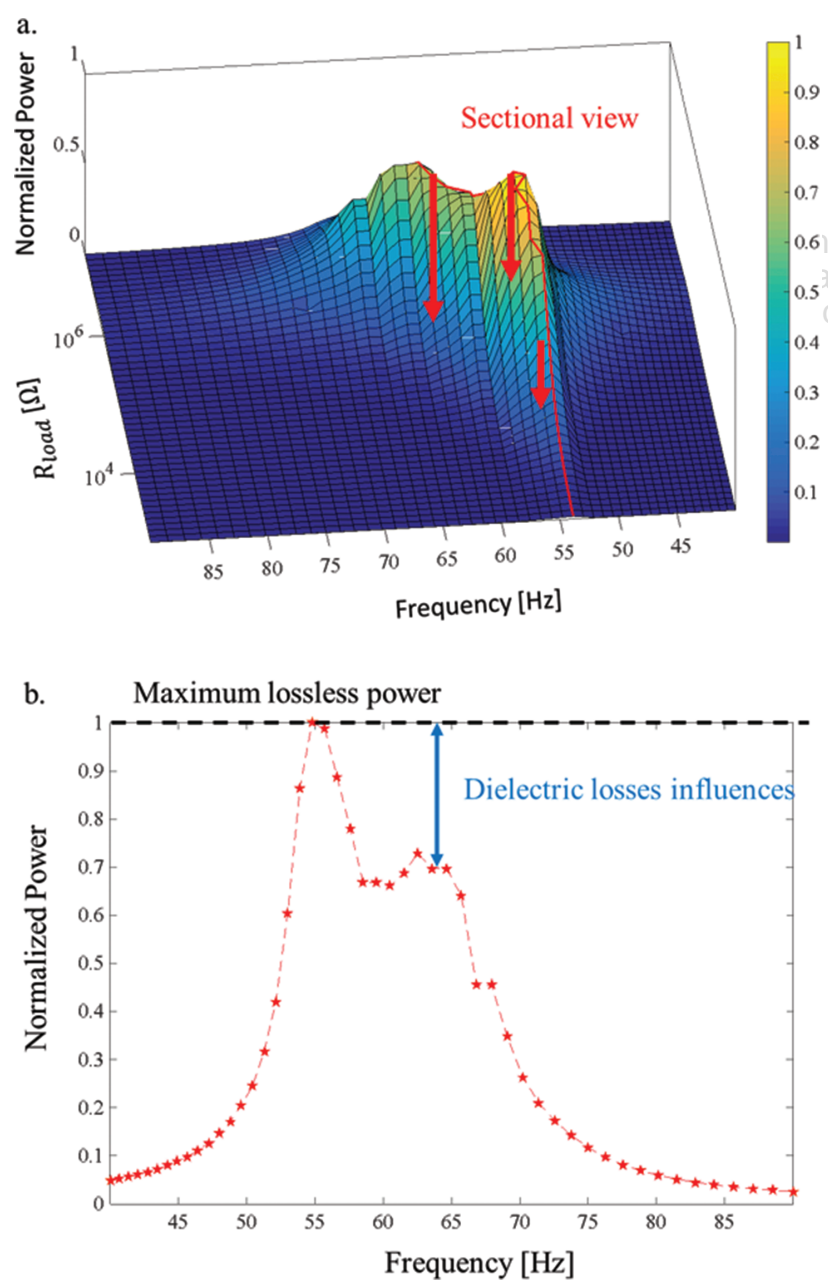

Fig. 16. Experimental harvested power (a) in the $\left(R_{\text {load }}, f\right)$ plane and (b) as a function of the frequency $f$ with an optimal $R_{\text {load }}$ maximizing the harvested power, with $\tan (\delta) \approx 10 \%$ and $\gamma=0.1 \mathrm{G}$. higher dielectric losses. Those losses can be observed from the differences of power between the two power peaks. Indeed, the dielectric losses do not affect the power around the short-circuit resonance frequency since the voltage level is quite low. However, as the excitation frequency gets closer to the PEH open-circuit resonance frequency, the voltage level increases, leading to higher dielectric losses. This behavior is consistent with the predicted one by the analytical model given by (19).

With further increase of the vibration magnitude, the dielectric losses become more and more important, as shown in Figure 16. This indeed confirms that dielectric losses become increasingly important with the electric field level, as expressed by (7), and shows how important it may be to take them into account.

\section{CONCLUSION}

In this paper, we first started by introducing the standard model of the dielectric losses in a piezoelectric material. We experimentally showed that the dielectric losses are dependent on the electric field across the piezoelectric material which explain why the dielectric losses effects can be observed while harvesting energy from vibrations (high electric field) even though they are very low in the manufacturer datasheets (low electric field). We derived energy boundaries for energy harvesting using linear piezoelectric harvesters, which take into account the losses in the dielectric material. Indeed, those losses set a fundamental harvested energy bandwidth limitation as it is impossible to tune the resonance frequency of the PEH without losing energy in the dielectric material. The influences of the dielectric losses have then been investigated on a simple energy harvesting interface (i.e., a purely resistive load) and the experimental results are in good agreement with the theoretical ones. Further work will focus on studying the dielectric losses influences while harvesting the energy using a non-linear electrical interfaces that exhibit higher piezoelectric voltages, such as Synchronous Electric Charges Extraction (SECE) and Synchronized Switch Harvesting on Inductor (SSHI) interfaces.

\section{References}

1. V. Raghunathan, A. Kansai, J. Hse, J. Friedman, and M. Srivastava, Design considerations for solar energy harvesting wireless embedded systems, Proceedings of the IPSN (2005), pp. 457-462.

2. A. Richelli, L. Colalongo, and K.-V. Zsolt, A $30 \mathrm{mV}-2.5 \mathrm{~V}$ DC/DC converter for energy harvesting. J. Low Power Electron. 11, 190 (2015).

3. G. Boccalero, C. Boragno, R. Morasso, and D. D. Caviglia, Efficiency issues for a wind-driven energy harvesting device. J. Low Power Electron. 14, 140 (2018).

4. D. Karolak, T. Taris, Y. Deval, J. B. Bégueret, and A. Mariano, Design of high sensitivity radiofrequency energy harvesters dedicated to low-power applications. J. Low Power Electron. 10, 72 (2014). 
5. Y. Wanderoild, A. Morel, A. Capitaine, and G. Pillonnet, A $50 \mu \mathrm{W}$ microbial fuel cell isolated energy harvesting interface based on air coupled inductors. J. Low Power Electron. 14, 170 (2018).

6. C. Knight, J. Davidson, and S. Behrens, Energy options for wireless sensor nodes. Sensors 8, 8037 (2008).

7. S. J. Roundy, Energy scavenging for wireless sensor nodes with a focus on vibration to electricity conversion, Ph.D. Thesis, University of California, Berkeley Berkeley, CA (2003).

8. S. Roundy, P. K. Wright, and J. Rabaey, A study of low level vibrations as a power source for wireless sensor nodes. Computer Communications 26, 1131 (2003).

9. E. Arroyo, A. Badel, F. Formosa, Y. Wu, and J. Qiu, Comparison of electromagnetic and piezoelectric vibration energy harvesters: Model and experiments. Sensors and Actuators A: Physical 183, 148 (2012).

10. G. K. Ottman, H. F. Hofmann, A. C. Bhatt, and G. A. Lesieutre, Adaptive piezoelectric energy harvesting circuit for wireless remote power supply. IEEE Transactions on Power Electronics 17, 669 (2002).

11. A. Nechibvute, A. Chawanda, and P. Luhanga, Piezoelectric energy harvesting devices: An alternative energy source for wireless sensors. Smart Materials Research 2012, Article ID 853481 (2012).

12. T.-B. Xu, E. J. Siochi, J. H. Kang, L. Zuo, W. Zhou, X. Tang, and X. Jiang, Energy harvesting using a PZT ceramic multilayer stack. Smart Mater. Struct. 22, 65015 (2013).

13. M.-G. Kang, W.-S. Jung, C.-Y. Kang, and S.-J. Yoon, Recent progress on PZT based piezoelectric energy harvesting technologies. Actuators 5, 5 (2016).

14. B. A. Seddik, G. Despesse, and E. Defay, Autonomous wideband mechanical energy harvester, 2012 IEEE International Symposium on Industrial Electronics (2012), pp. 1122-1127.

15. A. Morel, G. Pillonnet, and A. Badel, Regenerative synchronous electrical charge extraction for highly coupled piezoelectric generators, 2017 IEEE 60th International Midwest Symposium on Circuits and Systems (MWSCAS) (2017), pp. 237-240.

16. A. Badel and E. Lefeuvre, Wideband piezoelectric energy harvester tuned through its electronic interface circuit. Journal of Physics: Conference Series 557, 12115 (2014).

17. A. Morel, R. Grézaud, G. Pillonnet, P. Gasnier, G. Despesse, and A. Badel, Active AC/DC control for wideband piezoelectric energy harvesting. Journal of Physics: Conference Series 773, 12059 (2016).

18. A. Morel, A. Badel, P. Gasnier, Y. Wanderoild, and G. Pillonnet, Short circuit synchronous electric charge extraction (SC-SECE) strategy for wideband vibration energy harvesting, IEEE International Symposium of Circuits and Systems, (ISCAS) (2018), to be published.

19. S. Sherrit and B. K. Mukherjee, Characterization of piezoelectric materials for transducers, [Online]. Available: http:// arvix.org/ftp/arvix/papers/0711/0711.2657.pdf, November (2007).

20. M. W. Hooker, Properties of PZT-based piezoelectric ceramics between -150 and $250{ }^{\circ} \mathrm{C}$, National Aeronautics and Space Administration, Langley Research Center, Hampton, VA, USA (1998).

21. A. M. González, Á. García, C. Benavente-Peces, and L. Pardo, Revisiting the characterization of the losses in piezoelectric materials from impedance spectroscopy at resonance. Materials 9, 72 (2016).

22. Sinoceramics, Piezo Ceramics Specifications, Datasheet, Available: http://www.sinocera.net/en/piezo_material.asp (2017).

23. Sensor Technology, Piezoelectric Ceramics, Datasheet (2018).

24. Z. Yang and J. Zu, Comparison of PZN-PT, PMN-PT single crystals and PZT ceramic for vibration energy harvesting. Energy Conversion and Management 122, 321 (2016).

25. American Piezo, Physical and Piezoelectric Properties of APC Materials, Datasheet (2017).

26. K.-B. Kim, D. K. Hsu, B. Ahn, Y.-G. Kim, and D. J. Barnard, Fabrication and comparison of PMN-PT single crystal, PZT and PZTbased 1-3 composite ultrasonic transducers for NDE applications. Ultrasonics 50, 790 (2010).

27. A. Badel and E. Lefeuvre, Nonlinear conditioning circuits for piezoelectric energy harvesters, Nonlinearity Energy Harvest. Syst., edited by E. Blokhina, A. E. Aroudi, E. Alarcon, and D. Galayko, Springer International Publishing (2016), pp. 321-359.

28. N. G. Stephen, On the maximum power transfer theorem within electromechanical systems. Proceedings of the Institution of Mechanical Engineers, Part C: Journal of Mechanical Engineering Science 220, 1261 (2006)

29. J. Liang and W.-H. Liao, Impedance modeling and analysis for piezoelectric energy harvesting systems. IEEE/ASME Trans. Mechatronics 17, 1145 (2012).

30. A. Richter, A. Strobel, N. Joram, F. Ellinger, L. Gopfert, and R. Marg, Tunable interface for piezoelectric energy harvesting, Proc. 11th Int. Multi-Conf. on Systems, Signals and Devices (SSD) (2014).

31. H. Abdelmoula and A. Abdelkefi, Ultra-wide bandwidth improvement of piezoelectric energy harvesters through electrical inductance coupling. The European Physical Journal Special Topics 224, 2733 (2015).

32. Y. C. Shu and I. C. Lien, Analysis of power output for piezoelectric energy harvesting systems. Smart Mater. Struct. 15, 1499 (2006).

\section{A. Morel}

A. Morel was born in Valenciennes, France, in 1993. He received his electrical engineering degree from the National Institute of Applied Sciences of Lyon (INSA Lyon) and his M.Sc. degree in integrated systems from the University of Lyon, both in 2016. He is currently pursuing his Ph.D. at CEA-LETI in Grenoble, France. His research interests focus on micro-energy harvesting, multi-physics interactions, integrated power management, and ultra-low-power analog circuit design.

\section{G. Pillonnet}

G. Pillonnet was born in Lyon, France, in 1981. He received his Master's degree in Electrical Engineering from CPE Lyon, France, in 2004, a Ph.D. and habilitation degrees from INSA Lyon, France in 2007 and 2016, respectively. Following an early experience as analog designer in STMicroelectronics in 2008, he joined the University of Lyon in the Electrical Engineering department. During the 2011-12 academic year, he held a visiting researcher position at the University of California at Berkeley. Since 2013, he has been a full-time researcher at the CEA-LETI, a major French research institution. His research focuses on low-power electronics using heterogeneous devices including modeling, circuit design and control techniques. He has published more than 70 papers in his areas of interest. 


\section{Y. Wanderoild}

Y. Wanderoild was born in Grenoble, France, in 1991. He received his Master's of science in electronics, electrical energy and automated control as part of the ENS Paris Saclay degree in 2015. He is currently a Ph.D. candidate in the CEA-LETI, France. His research interests are focused on power electronic circuits and systems.

\section{A. Badel}

A. Badel graduated from Institut National des Sciences Appliquées de Lyon (INSA), Lyon, France, in electrical engineering in 2002 (M.S. degree). He prepared his Ph.D. at the Electrical Engineering and Ferroelectricity Laboratory of INSA Lyon, France. He received his Ph.D. degree in 2005 for his work on vibration control and energy harvesting. From November 2005 to November 2007, he was a JSPS (Japanese Society for the Promotion of Science) postdoctoral fellow at the Institute of Fluid Science of Tohoku University, Sendai, Japan. He is now a full professor at the Laboratory of Systems and Materials for Mechatronics from the Universite Savoie Mont Blanc, Annecy, France. His research interests include energy harvesting, vibration damping and piezoelectric actuators modeling and control. 